\title{
Article
}

\section{On further results of hex derived networks}

\author{
Haidar $\mathrm{Ali}^{1, *}$ and Ammara Sajjad ${ }^{2}$ \\ 1 Department of Mathematics, Government College University, Faisalabad, Pakistan. \\ 2 Government Public Girls High School, 214 RB, Faisalabad, Pakistan.; ammarakhan318@gmail.com \\ * Correspondence: haidarali@gcuf.edu.pk
}

Received: 18 October 2018; Accepted: 28 December 2018; Published: 21 March 2019.

\begin{abstract}
Topological indices are real numbers associated with molecular graphs of compounds that help to guess properties of compounds. Hex-Derived networks has an assortment of valuable applications in drug store, hardware, and systems administration. Imran et al. [1] computed the general Randić, first Zagreb, $\mathrm{ABC}, \mathrm{GA}, \mathrm{ABC}_{4}$, and $\mathrm{GA}_{5}$ indices for these hex-derived networks. In this article, we extend the work of [1] and compute some new topological indices of these networks.
\end{abstract}

Keywords: Irregularity index, Balaban index, Zagreb index, redefine Zagreb index.

MSC: 05C12, 05C90, 05C05.

\section{Introduction}

graph can be perceived by a polynomial, a numeric number, a matrix or a sequence of numbers. There are three types of topological indices:

1. degree-based

2. distance-based

3. counted related

Among these types, degree based indices are of incredible significance and assume an indispensable part in chemistry. The idea of chemical indices originated from Wiener [2]. The hexagonal mesh was put forward by Chen et al. [3]. A hexagonal work is made up with an arrangement of triangles as depicted in Figure 1. A 2-dimensional hexagonal mesh $H X(2)$ is made out of six triangles (see Figure 1(1)). By adding a layer of triangles around the boundary of $H X(2)$, a 3-dimensional hexagonal mesh $H X(3)$ is obtained (see Figure 1(2)).

In this article, $\mathcal{G}$ and $\mathcal{H}$ are considered to be Hex-Derived networks of type 1 and type 2, with vertex set $V$ and edge set $\mathrm{E}$. The notations used in this article are mainly taken from books [4,5].

Let $\mathfrak{T}$ be a graph. Then the Wiener index of $\mathfrak{T}$ is defined as

$$
W(\mathfrak{T})=\frac{1}{2} \sum_{(r, s)} d_{\mathfrak{T}}(r, s)
$$

where $(r, s)$ is any ordered pair of vertices in $\mathfrak{T}$ and $d(r, s)$ is $r-s$ geodesic.

The Zagreb indices discovered many applications in QSAR and QSPR reviews. In the books by Todeschini and Consonni [6,7], the details on the chemical applications of the two Zagreb indices can be found. If $d_{\mathfrak{T}}(r)$ be the degree of a vertex $r$ of a molecular simple connected graph $\mathfrak{T}$, the first and second Zagreb indices are defined as

$$
\begin{aligned}
& M_{1}(\mathfrak{T})=\sum_{r s \in E(\mathfrak{T})}\left(d_{\mathfrak{T}}(r)+d_{\mathfrak{T}}(s)\right), \\
& M_{2}(\mathfrak{T})=\sum_{r s \in E(\mathfrak{T})}\left(d_{\mathfrak{T}}(r) \cdot d_{\mathfrak{T}}(s)\right) .
\end{aligned}
$$

Hamzeh and Réti [8] concentrated on the Zagreb index disparity acquired from graph irregularity measures. For the essential delineation of a related graphs $\mathfrak{T}$ with $|V(\mathfrak{T})|$ vertices and $|E(\mathfrak{T})|$ edges, two novel graph 
irregularity indices are presented, which are depicted as follows.

Let $\mathfrak{T}$ be a graph with size ' $m$ ' and order ' $n$ ', then the first and second Zagreb irregularity indices are defined as

$$
\begin{gathered}
\operatorname{IRM}_{1}(\mathfrak{T})=M_{1}(\mathfrak{T})-\frac{4|E(\mathfrak{T})|^{2}}{|V(\mathfrak{T})|}, \\
\operatorname{IRM}_{2}(\mathfrak{T})=M_{2}(\mathfrak{T})-\frac{4|E(\mathfrak{T})|^{3}}{|V(\mathfrak{T})|^{2}} .
\end{gathered}
$$

Another topological index in view of the level of the vertex is the Balaban index $[9,10]$. This index for a graph $\mathfrak{T}$ of order ' $n$ ', size ' $m$ ' is characterized as

$$
J(\mathfrak{T})=\left(\frac{m}{m-n+2}\right) \sum_{r s \in E(\mathfrak{T})} \frac{1}{\sqrt{d_{\mathfrak{T}}(r) \cdot d_{\mathfrak{T}}(s)}} .
$$

Ghorbani and Azimi [11] defined the multiple Zagreb topological indices of a graph $\mathfrak{T}$ based on degree of vertices of $\mathfrak{T}$.

The first and second multiple Zagreb indices are defined as

$$
\begin{aligned}
& P M_{1}(\mathfrak{T})=\prod_{r s \in E(\mathfrak{T})}\left(d_{\mathfrak{T}}(r)+d_{\mathfrak{T}}(s)\right), \\
& P M_{2}(\mathfrak{T})=\prod_{r s \in E(\mathfrak{T})}\left(d_{\mathfrak{T}}(r) \cdot d_{\mathfrak{T}}(s)\right) .
\end{aligned}
$$

Properties of the first and second multiple Zagreb indices may be found in the work by Eliasi et al. [12] and Gutman [13]. Ranjini et al. [14] reclassified the Zagreb index and redefined Zagreb index as

$$
\operatorname{ReZG}(\mathfrak{T})=\sum_{r s \in E(\mathfrak{T})}\left(\frac{d_{\mathfrak{T}}(r)+d_{\mathfrak{T}}(s)}{d_{\mathfrak{T}}(r) \cdot d_{\mathfrak{T}}(s)}\right)
$$

Nowadays there is an extensive research activity on several important chemical indices and their variants. For further study of topological indices of various graph families see, [15-19]. In this paper, we aim to study the add.

\section{Discussion and Main results}

In this section, we present our main results. In section 2.1, we study hex derived network of type 1 and in the section 2.2, we study hex derived network of type 2 .

\subsection{Hex Derived network of type 1}

A planar graph $\mathfrak{T}$ partitions the rest of the plane into a number of arc wise-connected open sets, which are called the faces of $\mathfrak{T}$ [20]. If two faces are adjacent, then they have at least one common edge. Every plane graph has one and only one unbounded face, called the outer face. Figure 1(3) shows that $H X(2)$ has seven faces $f_{0}, f_{1}, \ldots, f_{6}$ where $f_{0}$ is the outer face and $f_{1}$ is adjacent to $f_{0}, f_{2}$ and $f_{6}$. In planar graph $\operatorname{HX}(n)$, suppose any arbitrary face $f$ is bijective to one vertex $f^{*}$ except the outer face. If $f^{*}$ is located in the face $f$ and we connect the vertices of $f$ with $f^{*}$, then we get $\operatorname{HDN}_{1}(n)$. Figure 2 demonstrates $H_{D N}(4)$. In this section, we calculate certain degree based topological indices of Hex Derived network of type 1. We compute the first and second Zagreb irregularity indices, Balaban index, multiple Zagreb indices and redefine Zagreb index for hex derived network of type 1.

Theorem 1. Let $(\mathcal{G})=H D N_{1}(n)$ be the hex derived network of type 1. Then

1. $I R M_{1}(\mathcal{G})=536-510 n+162 n^{2}+\frac{76-96 n}{7+3 n(-5+3 n)}$.

2. $\operatorname{IRM}_{2}(\mathcal{G})=6\left(146-199 n+81 n^{2}-\frac{18(-1+n)^{3}(-8+9 n)^{3}}{(7+3 n(-5+3 n))^{2}}\right)$. 


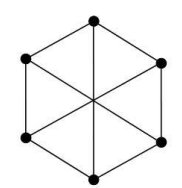

(1)

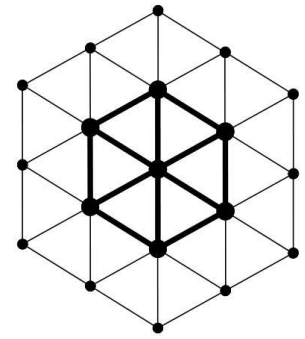

(2)

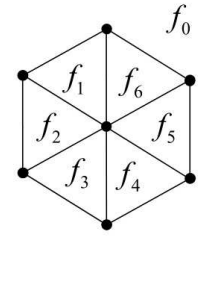

(3)

Figure 1. Hexagonal meshes: (1) HX(2) , (2) HX(3), and (3) all faces in $\mathrm{HX}(2)$.

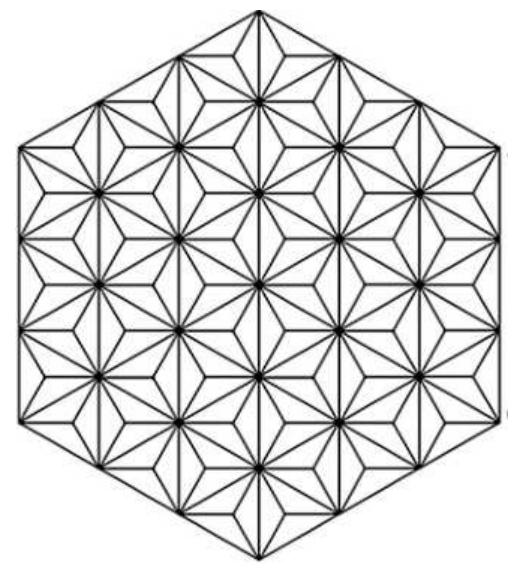

Figure 2. Hex Derived network $\left(H D N_{1}(4)\right)$

Proof. Let $\mathcal{G}=\operatorname{HDN}_{1}(n)$ be the hex derived network of type 1 , where $n \geq 4$. T $\mathcal{G}(n)$ has $6 n^{2}-12 n+6$ vertices of degree 3, 6 vertices of degree 5, $6 n-12$ vertices of degree 7 and $3 n^{2}-9 n+7$ vertices of degree 12 . Table 1 shows the different edge partitions of $\mathcal{G}(n)$.

(1) Now, from the definition of first Zagreb irregularity index, we have

$$
\begin{aligned}
I_{R} M_{1}(\mathcal{G})= & M_{1}(\mathcal{G})-\frac{4|E(\mathcal{G})|^{2}}{|V(\mathcal{G})|} \\
= & \sum_{r s \in \mathrm{E}(\mathcal{G})}\left(\text { degree }_{\mathcal{G}}(r)+\text { degree }_{\mathcal{G}}(s)\right)-\frac{4\left((3(-1+n)(-8+9 n))^{2}\right)}{1+3 n(-5+3 n)} \\
= & \mathrm{E}_{1}(8)+\mathrm{E}_{2}(10)+\mathrm{E}_{3}(15)+\mathrm{E}_{4}(12)+\mathrm{E}_{5}(17)+\mathrm{E}_{6}(14)+\mathrm{E}_{7}(19) \\
& +\mathrm{E}_{8}(24)-\frac{4\left((3(-1+n)(-8+9 n))^{2}\right)}{1+3 n(-5+3 n))} \\
= & (12)(8)+(18 n-36)(10)+\left(18 n^{2}-54 n+42\right)(15)+(12)(12) \\
& +(6)(17)+(6 n-18)(14)+(12 n-24)(19)+\left(9 n^{2}-33 n+30\right)(24) \\
& -\frac{4\left((3(-1+n)(-8+9 n))^{2}\right)}{1+3 n(-5+3 n))} \\
= & 536-510 n+162 n^{2}+\frac{76-96 n}{7+3 n(-5+3 n)} .
\end{aligned}
$$

(2) Now, from the definition of second Zagreb irregularity index, we have

$$
\begin{aligned}
\operatorname{IRM}_{2}(\mathcal{G}) & =M_{2}(\mathcal{G})-\frac{4|E(\mathcal{G})|^{3}}{|V(\mathcal{G})|^{2}} \\
& =\sum_{r s \in \mathrm{E}(\mathcal{G})}\left(d_{\mathcal{G}}(r)+d_{\mathcal{G}}(r)\right)-\frac{4\left((3(-1+n)(-8+9 n))^{3}\right)}{(1+3 n(-5+3 n))^{2}}
\end{aligned}
$$


Table 1. Edge partition of hex derived network $\operatorname{HDN}_{1}(n)$ based on degrees of end vertices of each edge.

\begin{tabular}{||cc||}
\hline$\left(d_{u}, d_{v}\right)$ where $u v \in E(\mathcal{H})$ & Number of edges \\
\hline \hline $\mathrm{E}_{1}=(3,5)$ & 12 \\
$\mathrm{E}_{2}=(3,7)$ & $18 n^{2}-54 n+42$ \\
$\mathrm{E}_{3}=(3,12)$ & 12 \\
$\mathrm{E}_{4}=(5,7)$ & 6 \\
$\mathrm{E}_{5}=(5,12)$ & $6 n-18$ \\
$\mathrm{E}_{6}=(7,7)$ & $12 n-24$ \\
$\mathrm{E}_{7}=(7,12)$ & $9 n^{2}-33 n+30$ \\
$\mathrm{E}_{8}=(12,12)$ &
\end{tabular}

$$
\begin{aligned}
= & \mathrm{E}_{1}(15)+\mathrm{E}_{2}(21)+\mathrm{E}_{3}(36)+\mathrm{E}_{4}(35)+\mathrm{E}_{5}(60)+\mathrm{E}_{6}(49)+\mathrm{E}_{7}(84) \\
& +\mathrm{E}_{8}(144)-\frac{4\left((3(-1+n)(-8+9 n))^{3}\right)}{(1+3 n(-5+3 n))^{2}} \\
= & (12)(15)+(18 n-36)(21)+\left(18 n^{2}-54 n+42\right)(36)+(12)(35)+ \\
& (6)(60)+(6 n-18)(49)+(12 n-24)(84)+\left(9 n^{2}-33 n+30\right)(144) \\
& -\frac{4\left((3(-1+n)(-8+9 n))^{3}\right)}{(1+3 n(-5+3 n))^{2}} \\
= & 6\left(146-199 n+81 n^{2}-\frac{18(-1+n)^{3}(-8+9 n)^{3}}{(7+3 n(-5+3 n))^{2}}\right) .
\end{aligned}
$$

Theorem 2. Let $(\mathcal{G})=H D N_{1}(n)$ be the hex derived network of type 1 . Then

$$
J(\mathcal{G})=\frac{3(-1+n)(-8+9 n)(970-280 \sqrt{2}+140 \sqrt{15}-240 \sqrt{21}+48 \sqrt{35}}{140(7+3 n(-5+3 n))}+\frac{5 n(-305+28 \sqrt{2}+24 \sqrt{21}+105 n))}{140(7+3 n(-5+3 n))}
$$

Proof. From the edge partition of $(\mathcal{G})=H D N_{1}(n)$ given in Table 1 , we have

$$
\begin{aligned}
J(\mathcal{G})= & \left(\frac{m}{m-n+2}\right) \sum_{r s \in \mathrm{E}(\mathcal{G})} \frac{1}{\sqrt{d_{\mathcal{G}}(r) \cdot d_{\mathcal{G}}(s)}} \\
= & \left(\frac{3(-1+n)(-8+9 n)}{7+3 n(-5+3 n)}\right)\left(\mathrm{E}_{1}\left(\frac{1}{\sqrt{3 \times 5}}\right)+\mathrm{E}_{2}\left(\frac{1}{\sqrt{3 \times 7}}\right)+\mathrm{E}_{3}\left(\frac{1}{\sqrt{3 \times 12}}\right)\right. \\
& \left.+\mathrm{E}_{4}\left(\frac{1}{\sqrt{5 \times 7}}\right)+\mathrm{E}_{5}\left(\frac{1}{\sqrt{5 \times 12}}\right)+\mathrm{E}_{6}\left(\frac{1}{\sqrt{7 \times 7}}\right)+\mathrm{E}_{7}\left(\frac{1}{\sqrt{7 \times 12}}\right)+\mathrm{E}_{8}\left(\frac{1}{\sqrt{12 \times 12}}\right)\right) \\
= & \left(\frac{3(-1+n)(-8+9 n)}{7+3 n(-5+3 n)}\right)\left((12)\left(\frac{1}{15}\right)+(18 n-36)\left(\frac{1}{21}\right)\right. \\
& +\left(18 n^{2}-54 n+42\right)\left(\frac{1}{36}\right)+(12)\left(\frac{1}{\sqrt{35}}\right)+(6)\left(\frac{1}{\sqrt{60}}\right)+(6 n-18)\left(\frac{1}{\sqrt{49}}\right) \\
& \left.+(12 n-24)\left(\frac{1}{\sqrt{84}}\right)+\left(9 n^{2}-33 n+30\right)\left(\frac{1}{\sqrt{144}}\right)\right) \\
& +\frac{3 n(-305+28 \sqrt{2}+24 \sqrt{21}+105 n))}{140(7+3 n(-5+3 n))} .
\end{aligned}
$$

Theorem 3. Let $(\mathcal{G})=H D N_{1}(n)$ be the hex derived network of type 1 . Then 
1. $P M_{1}(\mathcal{G})=12827693806929 \times 5^{18(-2+n)} 7^{6(-3+n)} 19^{12(-2+n)} 64^{1+4 n} 36^{18 n^{2}-54 n+42} 24^{9 n^{2}-33 n+30}$.

2. $P M_{2}(\mathcal{G})=931322574615478515625 \times 4^{6(-3+2 n)} 7^{42(-2+n)} 729^{-7+5 n} 36^{18 n^{2}-54 n+42} 144^{9 n^{2}-33 n+30}$.

Proof. (1) From the edge partition of $(\mathcal{G})=H D N_{1}(n)$ given in Table 1 and definition of multiple first Zagreb index, we have

$$
\begin{aligned}
P M_{1}(\mathcal{G})= & \prod_{r s \in \mathrm{E}(\mathcal{G})}\left(d_{\mathcal{G}}(r)+d_{\mathcal{G}}(s)\right) \\
= & 8^{\mathrm{E}_{1}(\mathcal{G})} \times 10^{\mathrm{E}_{2}(\mathcal{G})} \times 15^{\mathrm{E}_{3}(\mathcal{G})} \times 12^{\mathrm{E}_{4}(\mathcal{G})} \times 17^{\mathrm{E}_{5}(\mathcal{G})} \times 14^{\mathrm{E}_{6}(\mathcal{G})} \times 19^{\mathrm{E}_{7}(\mathcal{G})} \times 24^{\mathrm{E}_{8}(\mathcal{G})} \\
= & 8^{(12)}+10^{(18 n-36)}+15^{\left(18 n^{2}-54 n+42\right)}+12^{(12)}+17^{(6)}+14^{(6 n-18)}+19^{(12 n-24)}+24^{\left(9 n^{2}-33 n+30\right)} \\
= & 12827693806929 \times 5^{18(-2+n)} 7^{6(-3+n)} 19^{12(-2+n)} 64^{1+4 n} 36^{18 n^{2}-54 n+42} \\
& 24^{9 n^{2}-33 n+30}
\end{aligned}
$$

(2) Now, the definition of multiple second Zagreb index, we have

$$
\begin{aligned}
P M_{2}(\mathcal{G}) & =\prod_{r s \in \mathrm{E}(\mathcal{G})}\left(d_{\mathcal{G}}(r) \cdot d_{\mathcal{G}}(s)\right) \\
& =15^{\mathrm{E}_{1}(\mathcal{G})} \times 21^{\mathrm{E}_{2}(\mathcal{G})} \times 36^{\mathrm{E}_{3}(\mathcal{G})} \times 35^{\mathrm{E}_{4}(\mathcal{G})} \times 60^{\mathrm{E}_{5}(\mathcal{G})} \times 49^{\mathrm{E}_{6}(\mathcal{G})} \times 84^{\mathrm{E}_{7}(\mathcal{G})} \times 144^{\mathrm{E}_{8}(\mathcal{G})} \\
& =15^{(12)} \times 21^{(18 n-36)} \times 36^{\left(18 n^{2}-54 n+42\right)} \times 35^{(12)} \times 60^{(6)} \times 49^{(6 n-18)} \times 84^{(12 n-24)} \times 144^{\left(9 n^{2}-33 n+30\right)} \\
& =931322574615478515625 \times 4^{6(-3+2 n)} 7^{42(-2+n)} 729^{-7+5 n} 36^{18 n^{2}-54 n+42} 144^{9 n^{2}-33 n+30} .
\end{aligned}
$$

Theorem 4. Let $(\mathcal{G})=H D N_{1}(n)$ be the hex derived network of type 1 . Then

$$
\operatorname{ReZG}(\mathcal{G})=7+3 n(-5+3 n)
$$

Proof. From the edge partition of $(\mathcal{G})=H D N_{1}(n)$ given in Table 1 , we have

$$
\begin{aligned}
\operatorname{ReZG}(\mathcal{G})= & \sum_{r s \in E(\mathcal{G})}\left(\frac{d_{\mathcal{G}}(r)+d_{\mathcal{G}}(s)}{d_{\mathcal{G}}(r) \cdot d_{\mathcal{G}}(s)}\right) \\
= & \mathrm{E}_{1}\left(\frac{3+5}{3 \times 5}\right)+\mathrm{E}_{2}\left(\frac{3+7}{3 \times 7}\right)+\mathrm{E}_{3}\left(\frac{3+12}{3 \times 12}\right)+\mathrm{E}_{4}\left(\frac{5+7}{5 \times 7}\right)+\mathrm{E}_{5}\left(\frac{5+12}{5 \times 12}\right) \\
& +\mathrm{E}_{6}\left(\frac{7+7}{7 \times 7}\right)+\mathrm{E}_{7}\left(\frac{7+12}{7 \times 12}\right)+\mathrm{E}_{8}\left(\frac{12+12}{12 \times 12}\right) \\
= & (12)\left(\frac{8}{15}\right)+(18 n-36)\left(\frac{10}{21}\right)+\left(18 n^{2}-54 n+42\right)\left(\frac{5}{12}\right)+(12)\left(\frac{12}{35}\right) \\
& +(6)\left(\frac{17}{60}\right)+(6 n-18)\left(\frac{2}{7}\right)+(12 n-24)\left(\frac{19}{84}\right)+\left(9 n^{2}-33 n+30\right)\left(\frac{1}{6}\right) \\
= & 7+3 n(-5+3 n) .
\end{aligned}
$$

\subsection{Hex Derived network of type 2}

Assume that $f$ is adjacent to $f_{1}, f_{2}, \ldots, f_{k}$ and $f_{1}^{*}, f_{2}^{*}, \ldots, f_{k}^{*}$ are bijective to $f_{1}, f_{2}, \ldots, f_{k}$, respectively. If we join the vertices of $f$ and $f_{1}^{*}, f_{2}^{*}, \ldots, f_{k}^{*}$ with $f^{*}$, then we get $\operatorname{HDN}_{2}(n)$. Clearly, $\operatorname{HDN}_{1}(n)$ is a subgraph of $H_{D N}(n)$. Figure 3 shows $H_{D N}(4)$. In this section, we calculate certain degree based topological indices of Hex Derived network of type 2. We compute the first and second Zagreb irregularity indices, Balaban index, multiple Zagreb indices and redefine Zagreb index for hex derived network of type 2.

Theorem 5. Let $\mathcal{H}=\mathrm{HDN}_{2}(n)$ be Hex Derived network of type 2. Then 


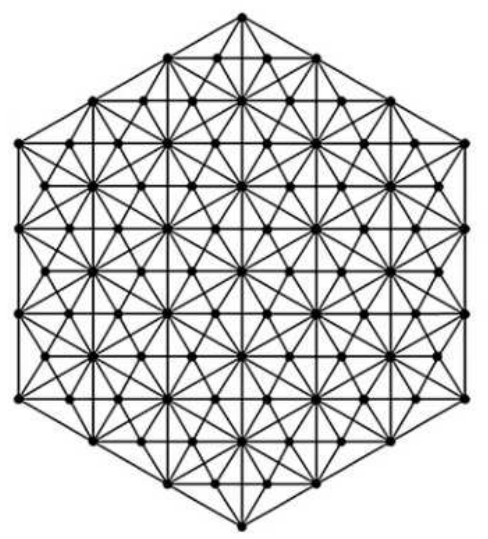

Figure 3. Hex Derived network $\left(\mathrm{HDN}_{2}(4)\right)$

Table 2. Edge partition of Hex Derived network $H_{D N}(n)$ based on degrees of end vertices of each edge.

\begin{tabular}{||cc||}
\hline$\left(d_{u}, d_{v}\right)$ where $u v \in E(\mathcal{H})$ & Number of edges \\
\hline \hline $\mathrm{E}_{1}=(5,5)$ & 18 \\
$\mathrm{E}_{2}=(5,6)$ & $12 n-24$ \\
$\mathrm{E}_{3}=(5,7)$ & $6 n$ \\
$\mathrm{E}_{4}=(5,12)$ & $9 n^{2}-33 n+30$ \\
$\mathrm{E}_{5}=(6,6)$ & $6 n-12$ \\
$\mathrm{E}_{6}=(6,7)$ & $18 n^{2}-60 n+48$ \\
$\mathrm{E}_{7}=(6,12)$ & $6 n-18$ \\
$\mathrm{E}_{8}=(7,7)$ & $12 n-24$ \\
$\mathrm{E}_{9}=(7,12)$ & $9 n^{2}-33 n+30$ \\
$\mathrm{E}_{10}=(12,12)$ &
\end{tabular}

1. $\operatorname{IRM}_{1}(\mathcal{H})=12\left(7-13 n+6 n^{2}-\frac{16(-1+n)}{7+3 n(-5+3 n)}\right)$.

2. $\operatorname{IRM}_{2}(\mathcal{H})=6\left(794+n(-1261+486 n)-\frac{31104\left(-1+n^{6}\right)}{(7+3 n(-5+3 n))^{2}}\right)$.

Proof. Suppose $\mathcal{H}=H_{D N}(n)$ be the Hex Derived network of type 2, where $n \geq 4$. $\mathcal{H}$ has $6 n$ vertices of degree $5,6 n^{2}-18 n+12$ vertices of degree $6,6 n-12$ vertices of degree 7 and $3 n^{2}-9 n+7$ vertices of degree 12. The edge set of $\mathcal{H}$ is divided into ten partitions based on the degree of end vertices. Table 2 shows the different edge partitions of $\mathcal{H}$. (1) Now from the definition of first Zagreb irregularity index, we have

$$
\begin{aligned}
\operatorname{IRM}_{1}(\mathcal{H})= & M_{1}(\mathcal{H})-\frac{4|E(\mathcal{H})|^{2}}{|V(\mathcal{H})|} \\
= & \sum_{r s \in E(\mathcal{H})}\left(d_{\mathcal{H}}(r)+d_{\mathcal{H}}(s)\right)-\frac{4\left(\left(36(-1-n)^{2}\right)^{2}\right)}{7+3 n(-5+3 n)} \\
= & E_{1}(10)+E_{2}(11)+E_{3}(12)+E_{4}(17)+E_{5}(12)+E_{6}(13)+E_{7}(18)+E_{8}(14)+E_{9}(19)+E_{10}(24) \\
& -\frac{4\left(\left(36(-1-n)^{2}\right)^{2}\right)}{(7+3 n(-5+3 n))} \\
= & (18)(10)+(12 n-24)(11)+(12 n-12)(12)+(6 n)(17)+\left(9 n^{2}-33 n+30\right)(12)+(6 n-12)(13) \\
& +\left(18 n^{2}-60 n+48\right)(18)+(6 n-18)(14)+(12 n-24)(19)+\left(9 n^{2}-33 n+30\right)(24) \\
& -\frac{4\left(\left(36(-1-n)^{2}\right)^{2}\right)}{(1+3 n(-5+3 n))} \\
= & 12\left(7-13 n+6 n^{2}-\frac{16(-1+n)}{7+3 n(-5+3 n)}\right)
\end{aligned}
$$


(2) Now, from the definition of second Zagreb irregularity index, we have

$$
\begin{aligned}
\operatorname{IRM}_{2}(\mathcal{H})= & M_{2}(\mathcal{H})-\frac{4|E(\mathcal{H})|^{3}}{|V(\mathcal{H})|^{2}} \\
= & \sum_{r s \in E(\mathcal{H})}\left(d_{\mathcal{H}}(r)+d_{\mathcal{H}}(s)\right)-\frac{4\left(\left(36(-1-n)^{2}\right)^{3}\right)}{7+3 n(-5+3 n)^{2}} \\
= & E_{1}(25)+E_{2}(30)+E_{3}(35)+E_{4}(60)+E_{5}(36)+E_{6}(42)+E_{7}(72)+E_{8}(49)+E_{9}(84)+E_{10}(144) \\
& -\frac{4\left(\left(36(-1-n)^{2}\right)^{3}\right)}{7+3 n(-5+3 n)^{2}} \\
= & (18)(25)+(12 n-24)(30)+(12 n-12)(35)+(6 n)(60)+\left(9 n^{2}-33 n+30\right)(36)+(6 n-12)(42) \\
& +\left(18 n^{2}-60 n+48\right)(72)+(6 n-18)(49)+(12 n-24)(84)+\left(9 n^{2}-33 n+30\right)(144) \\
& -\frac{4\left(\left(36(-1-n)^{2}\right)^{3}\right)}{7+3 n(-5+3 n)^{2}} \\
= & 6\left(794+n(-1261+486 n)-\frac{31104\left(-1+n^{6}\right)}{(7+3 n(-5+3 n))^{2}}\right)
\end{aligned}
$$

Theorem 6. Let $\mathcal{H}=H_{D} N_{2}(n)$ be Hex Derived network of type 2. Then

$J(\mathcal{H})=36(1+n)^{2}\left[\frac{\left(\frac{18}{5}+\frac{6}{7}(-3+n)+2 \sqrt{\frac{3}{7}}(-2+n)+2 \sqrt{\frac{6}{5}}(-2+n)\right)}{7+3 n(-5+3 n)}+\frac{\left(12(-1+n) \sqrt{35}+\sqrt{\frac{3}{15}} n+\frac{3}{4}(-2+n)(-5+3 n)+\frac{(-2+n)(-4+3 n)}{\sqrt{2}}\right)}{7+3 n(-5+3 n)}\right]$

Proof. From the edge partition given in Table 2 and definition of Balaban index, we have

$$
\begin{aligned}
& J(\mathcal{H})=\sum_{r s \in E(\mathcal{H})}\left(\frac{d_{\mathcal{H}}(r) \cdot d_{\mathcal{H}}(s)}{d_{\mathcal{H}}(r)+d_{\mathcal{H}}(s)-2}\right)^{3} \\
& =E_{1}\left(\frac{5 \times 5}{5+5-2}\right)^{3}+E_{2}\left(\frac{5 \times 6}{5+6-2}\right)^{3}+E_{3}\left(\frac{5 \times 7}{5+7-2}\right)^{3}+E_{4}\left(\frac{5 \times 12}{5+12-2}\right)^{3} \\
& +E_{5}\left(\frac{6 \times 6}{6+6-2}\right)^{3}+E_{6}\left(\frac{6 \times 7}{6+7-2}\right)^{3}+E_{7}\left(\frac{6 \times 12}{6+12-2}\right)^{3}+E_{8}\left(\frac{7 \times 7}{7+7-2}\right)^{3} \\
& +E_{9}\left(\frac{7 \times 12}{7+12-2}\right)^{3}+E_{10}\left(\frac{12 \times 12}{12+12-2}\right)^{3} \\
& =(18)\left(\frac{15625}{512}\right)+(12 n-24)\left(\frac{1000}{27}\right)+(12 n-12)\left(\frac{343}{8}\right)+(6 n)(64)+ \\
& \left(9 n^{2}-33 n+30\right)\left(\frac{5832}{125}\right)+(6 n-12)\left(\frac{74088}{1331}\right)+\left(18 n^{2}-60 n+48\right)\left(\frac{729}{8}\right) \\
& +(6 n-18)\left(\frac{117649}{1728}\right)+(12 n-24)\left(\frac{592704}{4913}\right)+\left(9 n^{2}-33 n+30\right)\left(\frac{373248}{1331}\right) \\
& =36(1+n)^{2}\left[\frac{\left(\frac{18}{5}+\frac{6}{7}(-3+n)+2 \sqrt{\frac{3}{7}}(-2+n)+2 \sqrt{\frac{6}{5}}(-2+n)\right)}{7+3 n(-5+3 n)}+\right. \\
& \left.\frac{\left(12(-1+n) \sqrt{35}+\sqrt{\frac{3}{15}} n+\frac{3}{4}(-2+n)(-5+3 n)+\frac{(-2+n)(-4+3 n)}{\sqrt{2}}\right)}{7+3 n(-5+3 n)}\right] .
\end{aligned}
$$

Theorem 7. Let $\mathcal{H}=\operatorname{HDN}_{2}(n)$ be Hex Derived network of type 2. Then

1. $P M_{1}(\mathcal{H})=38146972656252^{174+3 n(-65+21 n)} 3^{144+6 n(-29+9 n)} 7^{6(-3+n)} 13^{6(-2+n)} 17^{6 n} 209^{12(-2+n)}$. 
2. $P M_{2}(\mathcal{H})=2^{12(-5+3 n)(-4+3 n)} 3^{156+72(-3+n) n} 5^{30 n} 7^{42(-2+n)}$.

Proof. (1) From the edge partition given in Table 2 and definition of multiple first Zagreb index, we have

$$
\begin{aligned}
P M_{1}(\mathcal{H})= & \prod_{r s \in E(\mathcal{H})}\left(d_{\mathcal{H}}(r)+d_{\mathcal{H}}(s)\right) \\
= & 10^{E_{1}(\mathcal{H})} \times 11^{E_{2}(\mathcal{H})} \times 12^{E_{3}(\mathcal{H})} \times 17^{E_{4}(\mathcal{H})} \times 12^{E_{5}(\mathcal{H})} \times 13^{E_{6}(\mathcal{H})} \times 18^{E_{7}(\mathcal{H})} \\
& \times 14^{E_{8}(\mathcal{H})} \times 19^{E_{9}(\mathcal{H})} \times 24^{E_{10}(\mathcal{H})} \\
= & 10^{(18)} \times 11^{(12 n-24)} \times 12^{(12 n-12)} \times 17^{(6 n)} \times 12^{\left(9 n^{2}-33 n+30\right)} \times 13^{(6 n-12)} \\
& \times 18^{\left(18 n^{2}-60 n+48\right)} \times 14^{(6 n-18)} \times 19^{(12 n-24)} \times 24^{\left(9 n^{2}-33 n+30\right)} \\
= & 38146972656252^{174+3 n(-65+21 n)} 3^{144+6 n(-29+9 n)} 7^{6(-3+n)} 13^{6(-2+n)} \\
& 17^{6 n} 209^{12(-2+n)} .
\end{aligned}
$$

(2) Now from the definition of multiple second Zagreb index, we have

$$
\begin{aligned}
P M_{2}(\mathcal{H})= & \prod_{r s \in E(\mathcal{H})}\left(d_{\mathcal{H}}(r) \cdot d_{\mathcal{H}}(s)\right) \\
= & 25^{E_{1}(\mathcal{H})} \times 30^{E_{2}(\mathcal{H})} \times 35^{E_{3}(\mathcal{H})} \times 60^{E_{4}(\mathcal{H})} \times 36^{E_{5}(\mathcal{H})} \times 42^{E_{6}(\mathcal{H})} \times 72^{E_{7}(\mathcal{H})} \\
& \times 49^{E_{8}(\mathcal{H})} \times 84^{E_{9}(\mathcal{H})} \times 144^{E_{10}(\mathcal{H})} \\
= & 25^{(18)} \times 30^{(12 n-24)} \times 35^{(12 n-12)} \times 60^{(6 n)} \times 36^{\left(9 n^{2}-33 n+30\right)} \times 42^{(6 n-12)} \\
& \times 72^{\left(18 n^{2}-60 n+48\right)} \times 49^{(6 n-18)} \times 84^{(12 n-24)} \times 144^{\left(9 n^{2}-33 n+30\right)} \\
= & 2^{12(-5+3 n)(-4+3 n)} 3^{156+72(-3+n) n} 5^{30 n} 7^{42(-2+n)} .
\end{aligned}
$$

Theorem 8. Let $\mathcal{H}=\operatorname{HDN}_{2}(n)$ be Hex Derived network of type 2. Then

$$
\operatorname{ReZG}(\mathcal{H})=7+3 n(-5+3 n) .
$$

Proof. From the edge partition given in Table 2, we have

$$
\begin{aligned}
\operatorname{ReZG}(\mathcal{H})= & \sum_{r s \in E(\mathcal{H})}\left(\frac{d_{\mathcal{H}}(r)+d_{\mathcal{H}}(s)}{d_{\mathcal{H}}(r) \cdot d_{\mathcal{H}}(s)}\right) \\
= & \mathrm{E}_{1}\left(\frac{5+5}{5 \times 5}\right)+\mathrm{E}_{2}\left(\frac{5+6}{5 \times 6}\right)+\mathrm{E}_{3}\left(\frac{5+7}{5 \times 7}\right)+\mathrm{E}_{4}\left(\frac{5+12}{5 \times 12}\right)+\mathrm{E}_{5}\left(\frac{6+6}{6 \times 6}\right) \\
& +\mathrm{E}_{6}\left(\frac{6+7}{6 \times 7}\right)+\mathrm{E}_{7}\left(\frac{6+12}{6 \times 12}\right)+\mathrm{E}_{8}\left(\frac{7+7}{7 \times 7}\right)+\mathrm{E}_{9}\left(\frac{7+12}{7 \times 12}\right)+\mathrm{E}_{10}\left(\frac{12+12}{12 \times 12}\right) \\
= & (18)\left(\frac{2}{5}\right)+(12 n-24)\left(\frac{11}{30}\right)+(12 n-12)\left(\frac{12}{35}\right)+(6 n)\left(\frac{17}{60}\right) \\
& +\left(9 n^{2}-33 n+30\right)\left(\frac{1}{3}\right)+(6 n-12)\left(\frac{13}{42}\right)+\left(18 n^{2}-60 n+48\right)\left(\frac{1}{4}\right) \\
& +(6 n-18)\left(\frac{2}{7}\right)+(12 n-24)\left(\frac{19}{84}\right)+\left(9 n^{2}-33 n+30\right)\left(\frac{1}{6}\right) \\
= & 7+3 n(-5+3 n) .
\end{aligned}
$$

\section{Conclusions}

In this paper, we calculated different types of Zagreb and Balaban chemical indices for hex derived networks. These exact results are helpful in chemical point of view and in pharmaceutical sciences. We are looking forward in future to compute other chemical indices for hex derived networks. 


\section{Acknowledgements}

The authors would like to thank the respected reviewer for their suggestions and useful comments, which resulted in an improved version of this paper.

Author Contributions: All authors contributed equally to the writing of this paper. All authors read and approved the final manuscript.

Conflicts of Interest: "The authors declare no conflict of interest."

\section{References}

[1] Imran, M., Baig, A. Q., \& Ali, H. (2016). On molecular topological properties of hex̌̌derived networks. Journal of Chemometrics, 30(3), 121-129.

[2] Wiener, H. (1947). Structural determination of paraffin boiling points. Journal of the American Chemical Society, 69(1), 17-20.

[3] Chen, M. S., Shin, K. G., \& Kandlur, D. D. (1990). Addressing, routing, and broadcasting in hexagonal mesh multiprocessors. IEEE Transactions on Computers, 39(1), 10-18.

[4] Diudea, M. V., Gutman, I., \& Jantschi, L. (2001). Molecular topology. Huntington, NY: Nova Science Publishers.

[5] Gutman, I., \& Polansky, O. E. (2012). Mathematical concepts in organic chemistry. Springer Science \& Business Media.

[6] Todeschini, R., \& Consonni, V. (2008). Handbook of molecular descriptors (Vol. 11). John Wiley \& Sons.

[7] Todeschini, R., \& Consonni, V. (2009). Molecular descriptors for chemoinformatics: volume I: alphabetical listing/volume II: appendices, references (Vol. 41). John Wiley \& Sons.

[8] Hamzeh, A., \& RÃ̃lti, T. (2014). An analogue of Zagreb index inequality obtained from graph irregularity measures. MATCH Commun. Math. Comput. Chem, 72(3), 669-683.

[9] Balaban, A. T. (1982). Highly discriminating distance-based topological index. Chemical Physics Letters, 89(5), $399-404$.

[10] Balaban, A. T., \& Quintas, L. V. (1983). The smallest graphs, trees, and 4-trees with degenerate topological index. J. MATCH (Commun. Math. Chem.), 14, 213-233.

[11] Ghorbani, M., \& Azimi, N. (2012). Note on multiple Zagreb indices. Iranian Journal of Mathematical Chemistry, 3(2), 137-143.

[12] Eliasi, M., Iranmanesh, A., \& Gutman, I. (2012). Multiplicative versions of first Zagreb index. Match-Communications in Mathematical and Computer Chemistry, 68(1), 217.

[13] Gutman, I. (2011). Multiplicative Zagreb indices of trees. Bull. Int. Math. Virt. Inst, 1, 13-19.

[14] Ranjini, P. S., Lokesha, V., \& Usha, A. (2013). Relation between phenylene and hexagonal squeeze using harmonic index. International Journal of Graph Theory, 1(4), 116-121.

[15] Bača, M., Horváthová, J., Mokrišová, M., \& Suhányiovă, A. (2015). On topological indices of fullerenes. Applied Mathematics and Computation, 251, 154-161.

[16] Baig, A. Q., Imran, M., \& Ali, H. (2015). On topological indices of poly oxide, poly silicate, DOX, and DSL networks. Canadian Journal of chemistry, 93(7), 730-739.

[17] Imran, M., Baig, A. Q., \& Ali, H. (2015). On topological properties of dominating David derived networks. Canadian Journal of Chemistry, 94(2), 137-148.

[18] Liu, J. B., Ali, H., Shafiq, M., \& Munir, U. (2018). On Degree-Based Topological Indices of Symmetric Chemical Structures. Symmetry, 10(11), 619.

[19] Simonraj, F., \& George, A. (2012). Embedding of poly honeycomb networks and the metric dimension of star of david network. International Journal on Applications of Graph Theory in Wireless Ad Hoc Networks and Sensor Networks, 4(4), 11.

[20] Bondy, J. A., \& Murty, U. S. R. (1976). Graph theory with applications (Vol. 290). London: Macmillan.

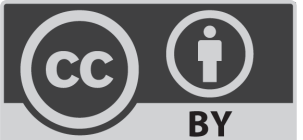

(C) 2019 by the authors; licensee PSRP, Lahore, Pakistan. This article is an open access article distributed under the terms and conditions of the Creative Commons Attribution (CC-BY) license (http://creativecommons.org/licenses/by/4.0/). 